\title{
Appareil
}

$10 \mid 2012$

Lyotard et la surface d'inscription numérique

\section{Le sublime, la surface picturale et les disparitions politiques}

Sur la polémique Rancière/Lyotard

Denis Skopin

\section{OpenEdition}

\section{Journals}

Édition électronique

URL : http://journals.openedition.org/appareil/985

DOI : 10.4000/appareil.985

ISSN : 2101-0714

Éditeur

MSH Paris Nord

\section{Référence électronique}

Denis Skopin, "Le sublime, la surface picturale et les disparitions politiques », Appareil [En ligne], 10 |

2012, mis en ligne le 20 décembre 2012, consulté le 10 décembre 2020. URL : http://

journals.openedition.org/appareil/985; DOI : https://doi.org/10.4000/appareil.985

Ce document a été généré automatiquement le 10 décembre 2020.

\section{c) (1) $\Theta \Theta$}

Appareil est mis à disposition selon les termes de la Licence Creative Commons Attribution - Pas d'Utilisation Commerciale - Pas de Modification 4.0 International. 


\title{
Le sublime, la surface picturale et les disparitions politiques
}

\author{
Sur la polémique Rancière/Lyotard
}

Denis Skopin

1 Grosso modo, la lecture de l'esthétique kantienne par Lyotard est construite sur l'interprétation des trois notions : celle de "passage » (Übergang) entre les facultés, celle de sensus communis (der gemeinschaftliche Sinn) et finalement celle de sublime. Premièrement, Lyotard déconstruit les passages interfacultaires chez Kant lui-même (illusion transcendantale, symbole, type, monogramme) pour protéger la philosophie kantienne contre sa transformation en système et isoler les facultés par un «abîme infranchissable ». La question qui se pose est la suivante : pourquoi Lyotard considèret-il comme des passages les quatre cas mentionnés ci-dessus ? Comment se fait-il qu'il aille à contre-courant de la tradition philosophique qui, depuis Schiller, veut que le passage entre l'entendement et la raison, leur connexion au sein de la faculté de juger, soit assuré par le jugement du goût? Tout d'abord, aux yeux de Lyotard, le passage n'est pas un simple "pont» reliant les deux facultés mais le transfert des règles d'une faculté dans l'autre, l'application des règles d'une faculté à «l'intérieur " de l'autre; ensuite, selon Lyotard, le jugement du goût n'est pas un passage. On se trompe en affirmant que les facultés s'unissent dans le jugement du goût; nous ressentons tout au plus la possibilité de leur « euphonie » dans le sujet. Cela signifie que sensus communis ne sera pas un accord empirique à propos du beau mais un signe ou un témoin de la compatibilité, toujours en puissance, des facultés dans chaque individu. Cette deuxième démarche qu'est la lecture du sensus communis a pour but de prévenir son interprétation empirique qui est déclarée coupable de tous les totalitarismes et les crimes politiques de la modernité. La troisième démarche, qui est la plus importante, consiste dans la relecture du sublime kantien.

2 Le sentiment sublime est un sentiment complexe qui est provoqué chez le spectateur par le jeu des opérations de Vorstellung et surtout de Darstellung, terme dont la difficulté pour la traduction est équivalente à son importance pour toute l'esthétique postkantienne. Il paraît que, mutatis mutandis, Lyotard identifie la Darstellung à la 
représentation, ce qui, en actualisant au maximum la fonction de visualisation de la Darstellung, permet à Lyotard de l'incorporer dans son opposition figural/ blocs d'écriture.

3 Si l'opération de Vorstellung s'applique aux objets de nature (an Naturobjekten), la Darstellung est une technique mentale plus délicate qui associe «dans l'esprit» (ins Gemüt) les concepts ou les idées avec des «images» correspondantes, ou plus précisément qui met ces images «à côté» des concepts (dem Begriffe eine korrespondierende Anschauung zur Seite stellt) ${ }^{1}$. La Darstellung n'est donc pas un état mais un processus, l'activité de l'imagination. Si le beau est compatible avec l'imagination « à l'œuvre ", le sentiment sublime est dû à une espèce de paralysie de celle-ci. Par suite d'un choc sensible, l'opération de Beförderung (du transport des "forces vitales", der Lebenskräfte), qui est au cœur de la Darstellung kantienne, est violemment interrompue et privée de son caractère systématique. Le flux sensible n'est plus systématisé ni contrôlé, il nous arrive tel quel, «brut », sans forme. Le sublime est toujours à trouver dans un objet infini ou informe (unendlich, formlos) dont la Darstellung ne peut être que négative (eine Darstellung des Unendlichen, welche zwar eben darum niemals anders als bloß negative Darstellung sein kann) ${ }^{2}$.

4 À l'instar de Kant, Lyotard fait un usage éthique de cette représentation négative. Elle portera le témoignage de l'impossibilité de représenter une faculté dans l'autre et se fera une alternative à la réunion des facultés. Si pour Kant le sentiment sublime fonctionnait comme signe d'histoire et alternative à la terreur des jacobins, Lyotard emprunte à Kant cette notion pour faire face à l'expérience totalitaire de la modernité : la Shoah ou la terreur de l'État stalinien. L'expérience de l'inhumain est irreprésentable comme telle, elle est incommensurable aux moyens de la représentation. Tout témoignage de cette expérience est un témoignage négatif. La tâche de témoigner, de repérer le "signe d'histoire » est attribuée par Lyotard à l'art « sublime », c'est-à-dire à l'art abstrait qui renonce à la figuration ${ }^{3}$.

5 La position de Rancière face au sublime est toute contraire. Dans son ouvrage Malaise dans l'esthétique, il déconstruit l'interprétation lyotardienne de Kant (le texte de Lyotard Après le sublime, état de l'esthétique dans Inhumain) et propose sa contre-lecture de la Troisième critique. Pour un schillerien qu'est Rancière, l'art est un moyen qui, comme le dit Schiller dans ses Lettres sur l'éducation esthétique de l'homme, permet de " réparer » le dispositif social sans l'arrêter (en d'autres mots, l'expérience esthétique chez Schiller va de pair avec l'expérience politique et même révolutionnaire). C'est pourquoi Rancière parle de la politique en termes esthétiques. Faire de la politique, c'est apparaître sur la scène politique, c'est, pour les sans-parts, franchir la limite qui les sépare de ceux qui sont esthétiquement - et par conséquent politiquement - visibles. D'après Rancière, le sublime au sens lyotardien ne peut pas avoir lieu dans la situation contemporaine, et le régime esthétique, qui s'est déjà établi dans la prose flaubertienne, est parfaitement adapté à la représentation de l'inhumain. Selon lui, il n'y a pas d'expérience d'exception, il n'y a pas d'expérience qu'on ne puisse pas inscrire aujourd'hui car les cadres du "régime esthétique » sont larges comme jamais. Si l'œdipe de Sophocle, plus précisément la scène nous montrant đEdipe en train de se crever les yeux, une scène dont la cruauté est exceptionnelle, se trouve irreprésentable sur les planches aux temps de Corneille, il ne l'est plus aujourd'hui. L'incommensurabilité entre l'expérience et sa représentation n'existe que du côté de l'expérience car dans la 
situation contemporaine les moyens de la représentation dépassent largement toute expérience, même la plus extrême ${ }^{4}$.

6 Ainsi, les positions de Rancière et Lyotard face au sublime constituent deux pôles opposés. D'un côte, l'échec de l'inscription du choc sensible avec le glissement dans la répétition de l'irreprésentable jusqu'à l'infini (Lyotard); de l'autre côté, le point de vue de Rancière qui, en montrant que n'importe quelle expérience peut être mise en paroles, dénie toute résistance de la matière à sa mise en forme. D'un côté, la représentation se révèle impossible; de l'autre côté, elle est déclarée sans entraves : selon Rancière, il n'y a aucun obstacle ni conditions techniques entre le sensible et sa représentation. D'un côté, la preuve nous échappe toujours et on ne peut témoigner de l'inhumain que négativement; de l'autre côté, le problème des preuves, du témoignage et de sa difficulté est déclaré peu actuel.

7 Afin de mieux comprendre les enjeux de cette polémique et, dans la mesure du possible, approcher la question du rôle du sentiment sublime pour la modernité esthétique et politique, je ne retiendrai qu'un seul thème qui nous servira de "fil conducteur ", à savoir le thème de lieu d'inscription ou de surface picturale.

Si l'on met à part sa démarche dans les Immatériaux, l'idée post-structuraliste de l'irréductibilité de l'image à la narration constitue le pivot des reflexions de Lyotard sur l'esthétique. Ce que veut faire Lyotard dans Discours, figure, c'est renverser l'équilibre entre le discursif et le figural au profit de l'image ou, comme il dit lui-même, « défendre l'œil ». Par son caractère indéchiffrable et opaque, «l'image » chez Lyotard ne diffère pas du punctum barthésien. Ensuite, ce que fait Lyotard dans Les Dispositifs pulsionnels et en particulier L'économie libidinale, c'est identifier l'absence de signifiance, le degré zéro de surface d'inscription à l'absence de profondeur. Cette deuxième idée résulte en quelque sorte de la première. Si la perspective est un système de signes, une écriture, l'art qui veut retrouver sa pureté, qui veut rompre avec son asservissement au discours doit par définition rompre avec l'appareil perspectif. Il s'en déduit que la peinture qui veut regagner sa pureté doit être par excellence une peinture qui regagne en planéité ou, ce qui est la même chose, qui s'oppose à l'évanouissement de son support. Sur ce point là, Lyotard rejoint l'idée greenbergienne de la révolution picturale comme découverte par la peinture de sa surface propre. Plus largement, c'est un discours qui insiste sur la primordialité du médium, qui veut penser l'autonomie de chaque art à partir de son support propre. Le support, la matérialité de l'œuvre est pensée par Lyotard comme une espèce de résistance aux codes "immatériaux», aux dispositifs d'aliénation dont la perspective fournit un exemple.

9 Lyotard pense la signifiance comme l'engendrement du volume tridimensionnel : faire signifier les objets, les représenter, c'est les faire entrer dans un système de valeurs qui est le système capitaliste. Celui-ci les fait fonctionner comme marchandises. Cet engendrement du volume théâtral est une pratique d'aliénation, une activité qui consiste à coder un événement. Pour le Lyotard de l'Économie libidinale, attaquer le capitalisme, c'est attaquer l'espace à trois dimensions et l'espace «théâtral » comme son modèle. Le théâtre est un dispositif, un transformateur qui capte l'énergie libidinale. C'est un dispositif qui fonctionne au même titre que la perspective, la tonalité musicale ou l'écriture, mais qui est en quelque sorte leur modèle, car le dédoublement théâtral, le passage au tridimensionnel est un processus constitutif de tout dispositif. L'apparition de la profondeur est inséparable de la transformation de l'énergie libidinale en marchandise. Cette énergie commence à fonctionner sous forme 
d'un travail cristallisé. Si les dispositifs dont il s'agit sont inséparables du système capitaliste, L'Économie libidinale cherche à décrire un état pré-capitaliste, un espace préthéâtral qui se caractérise par absence de profondeur. C'est un support pur et plan, un espace sans extériorité qui a pour modèle la bande de Möbius, surface, peau ou pellicule.

Contrairement à ce qu'on trouve chez Lyotard, dans l'esthétique rancièrienne l'image et la narration ne sont jamais séparés. Pour Rancière, il n'y a pas de peinture « muette » : il faut renoncer à l'idée de l'image « conçue comme une toile de Véronique où s'imprimerait le visage originaire des $\operatorname{choses}^{5} »$. Deuxièmement, le visible acquiert sa visibilité grâce aux paroles : ce sont les mots qui configurent les régimes de la visibilité. Pour qu'un tableau soit plan, il faut qu'il soit vu comme plan. Pourquoi le thème de la surface est-il important aux yeux de Rancière? La surface est le lieu où se localise le problème de l'articulation entre la narration et le visible. Proposer une contre-lecture de ce thème, c'est mettre en cause l'idée de l'indépendance de l'art de la littérature, l'idée de l'autonomisation de chaque art à partir de son support propre. La cible principale du texte la Surface du design est Clement Greenberg mais c'est aussi Lyotard dans la mesure où celui-ci reste fidèle à la conception de la révolution picturale comme conquête par la peinture de sa surface propre.

11 Rancière montre que le principe ut pictura poesis / ut poesis pictura est universel. Il ne règle pas seulement une période concrète de la co-existence des mots et des images, l'âge des Académies et des hiérarchies des genres. Les mots et les images sont toujours articulés : ce qui change, c'est la façon dont ils le sont. Cette articulation est flottante, elle varie selon les époques. Rancière montre que la planéité de la peinture n'a pas été, depuis l'âge symboliste jusqu'aux années 1920, un moyen de rupture avec l'asservissement au discours; au contraire, la surface a fonctionné comme un lieu où fait défaut toute hiérarchie, comme ce qui ôte aux mots et aux images leur propre. Les images y commencent à fonctionner comme textes et les textes comme images. "La surface revendiquée comme le medium propre de la pure peinture est en fait un autre medium. Elle est le théâtre d'une défiguration/dénomination ${ }^{6} »$. La surface constitue une limite où s'efface toute différence entre image et discours, où ils commencent à fonctionner en tant que signes : plus on est proche de cette limite (plus un tableau est plan), plus les images et les mots sont proches (degré zéro d'hiérarchies, on entre dans l'indistinction démocratique). Plus un tableau gagne en tridimensionnalité, plus les mots et les images sont séparés, plus leurs rapports sont hiérarchisés.

Pour illustrer cette divergence, on peut confronter les lectures du Coup de dés de Stéphane Mallarmé que font Rancière et Lyotard. Selon Lyotard, la tâche de Mallarmé en tant que poète consiste à débarrasser le langage articulé de sa fonction utilitaire et prosaïque, c'est-à-dire de sa fonction de communication. D'abord, Mallarmé rompt avec la prosodie traditionnelle, avec la structure mélodique du vers. Mais cela ne suffit pas pour rompre avec le côté fonctionnel du langage : il serait absurde de dire que les mots du poème ne signifient rien, qu'ils sont dénués de sens et n'accomplissent pas sa fonction « communicative ». Si obscure qu'il soit, le texte du poème mallarméen se lit; comme n'importe quel texte il a un référent. Cela signifie que l'espace du poème se trouve dédoublé, il devient, comme tout espace discursif, un espace à trois dimensions. C'est pourquoi la nouveauté du poème réside moins dans ses performances discursives (figures de style) que dans sa distribution typographique qui constitue une forme de rupture avec le discours. La matérialité des éléments graphiques et plastiques 
bouleverse l'immatérialité de l'agencement textuel du poème. Le désir de Mallarmé de donner aux mots, s'aidant des blancs typographiques, une structure spatiale, parfois même la forme des objets qu'ils désignent, n'est pas naiff : il témoigne de la même rotation du signifié qui devient le désigné qu'on trouve dans l'œuvre de Mazaccio. On assiste à une révolution poétique qui va de pair avec la révolution politique et picturale. Dans la mesure où il gagne en planéité, le poème de Mallarmé perd son caractère de poème et s'approche d'un tableau moderniste. La surface est affirmée par Lyotard comme un lieu où la figure acquiert de l'autonomie par rapport au discours, où la peinture s'émancipe par rapport à l'écriture. La blancheur de la page dans sa matérialité muette est ce qui résiste au discours ${ }^{7}$.

Dans la Surface du design, la démarche de Rancière consiste à mettre en parallèle l'activité poétique de Mallarmé et l'activité du designer Peter Behrens. Mallarmé n'est pas un poète qui s'enferme dans sa tour d'ivoire où il produit des poèmes hermétiques, et qui renonce à connaître la prose de la vie. Au contraire, ses recherches poétiques sont en conformité avec les recherches artistiques de ses contemporains, et en général avec l'esprit de son époque qui veut supprimer toute frontière entre les formes de la vie et les formes de l'art. Si Mallarmé veut donner à son poème une structure spatiale, ce n'est pas pour que celui-ci perde son caractère de poème et l'image perce derrière l'agencement textuel. Au contraire, l'image et le texte chez lui sont inséparables, le blanc typographique est un procédé aussi textuel que pictural, c'est un signe. De la même façon, Mallarmé veut constituer une poétique commune au poème et à l'activité chorégraphique. Si Mallarmé valorise le ballet contre le théâtre, s'il veut rompre avec la profondeur théâtrale, ce n'est pas parce qu'il veut rompre avec l'écriture mais, tout au contraire, parce que pour lui le ballet, tout comme poème ou tableau, est un type d'écriture. On trouve la même préoccupation de lever la barrière entres les arts chez Tchurlionis qui veut constituer un tableau comme partition. C'est aussi le cas de Behrens pour qui les formes des objets s'approchent des signes. Dans tous ces cas il s'agit de penser les arts comme jeu de signes. La surface est alors ce qui ôte aux arts leur propre. La surface ne fonctionne pas comme médium qui permet à la peinture de ne pas parler, d'obtenir son indépendance par rapport à la parole ou à l'écriture. C'est une interface, un lieu où disparaitt tout écart entre l'image, le texte et la note musicale qui commencent à fonctionner en tant que signes ${ }^{8}$.

14 La surface picturale pure et plane, témoigne-t-elle de l'irreprésentable, garant de la sublimité de l'art contemporain et protégeant celui-ci contre sa transformation en marchandise? Est-elle la gardienne éthique des identités et de l'impossibilité de leur communication? Ou, au contraire, elle est un lieu où culmine la représentation, un lieu d'indistinction qui met entre parenthèses toutes les hiérarchies et limites séparant les arts et les identités?

15 Je tenterai de répondre à ces questions à travers l'analyse de plusieurs photos que j'ai trouvées dans les archives de ma ville. Dans la plupart des cas, il s'agit de photos de groupe où sont représentés les leaders du Parti communiste russe dans les années 1930. Lors des purges staliniennes, certains visages - ceux des «ennemis du peuple» - ont été noircis à l'encre, rayés, grattés ou tout simplement découpés avec des ciseaux. Cette pratique, très courante à l'époque, ne coïncide pas du tout avec la censure. Elle constitue un phénomène beaucoup plus complexe et terrifiant car, dans la plupart des cas, c'étaient les proches de la victime qui faisaient disparaître sa représentation de cette façon. Le plus souvent, les visages noircis appartiennent aux anciens bolchéviks 
qui ont activement pris part à la Révolution. C'est pourquoi le noircissement des photos est inséparable de la falsification de l'histoire de la Révolution et le caviardage des noms des fusillés dans les livres et les manuels. La réécriture de l'histoire ne se faisait pas aux cabinets des historiens mais sur place, au cours des leçons d'histoire, quand les élèves, suivant les instructions de leur professeur, rayaient dans les manuels les noms des « ennemis du peuple». Dans les années 1930, la présence permanente d'un leader politique est souvent substituée par son absence inopinée: pour expliquer cette transformation subite la propagande stalinienne forge toute une panoplie de termes qui assurent la «dialectique » paranoïaque métamorphosant l'ami du peuple en son ennemi : déguisement, conspiration, sabotage etc., qui sont inévitablement suivis par le dévoilement et la punition. Cette économie bien huilée de la transformation des amis en ennemis n'a besoin de l' "iconoclasme sélectif» pratiqué sur les photos des victimes. La privation du visage n'a tout son sens que si on l'inscrit dans une autre logique : celle de l'effacement des traces d'un crime politique, nommée « la politique de la disparition ${ }^{9}$.

Le montage photographique fait par A. Ivanov dans l'esprit d'un constructivisme tardif doit représenter un événement important: inauguration du congrès des oudarniks (travailleurs de choc) travaillant dans le bâtiment ${ }^{10}$.

Figure 1

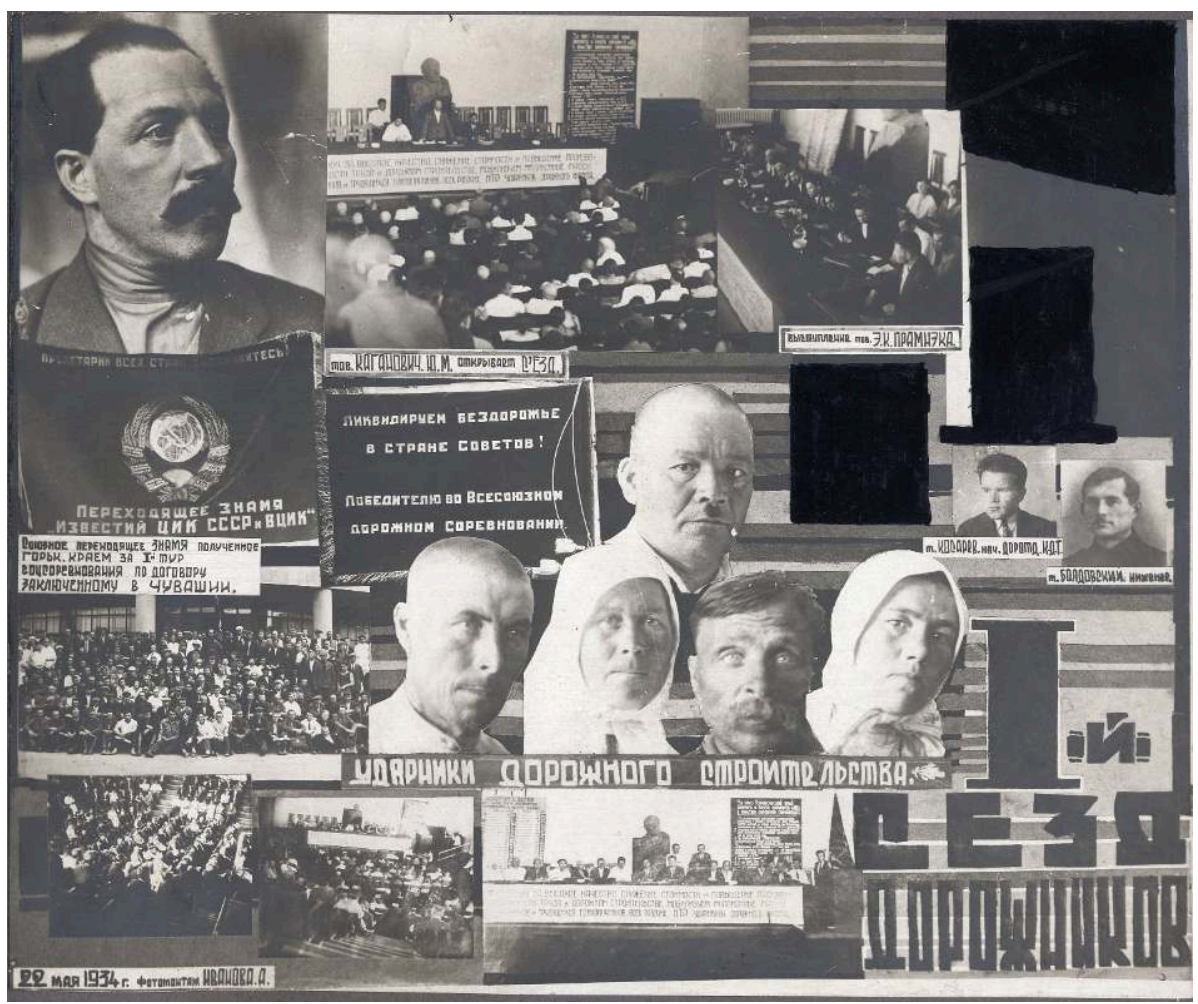

La partie droite de la photo a été noircie à l'encre. Il est fort probable que ce caviardage visait le premier secrétaire du Parti bolchévik à Nijni-Novgorod E. Pramnek qui a été fusillé en 1938. À cause des trous béants dans son espace organique, cette photographie a un aspect mutilé. Sur une autre photo ${ }^{11}$ sont représentés les " oudarniks d'honneur » de la construction d'un pont à Nijni-Novgorod. 


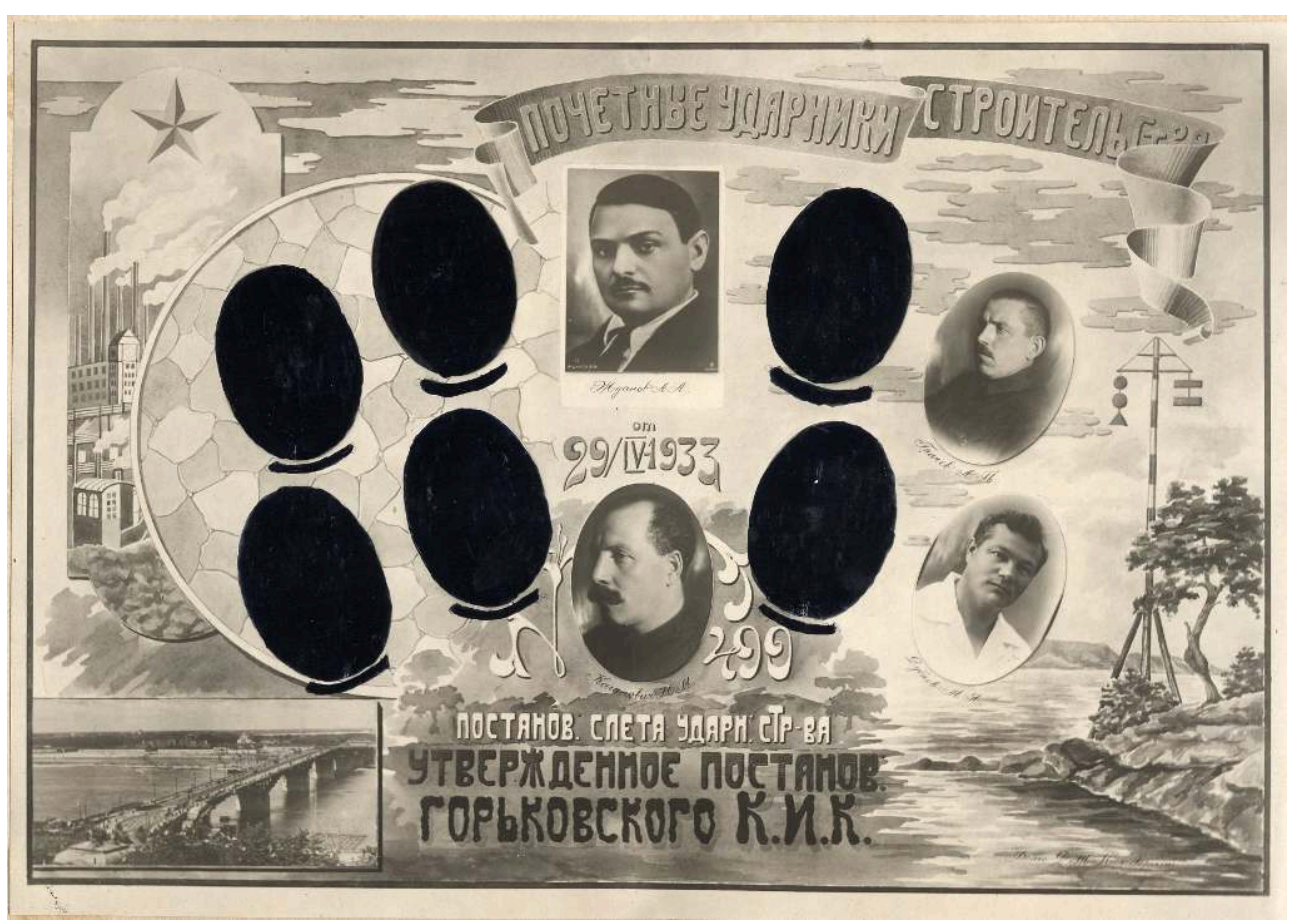

Il s'agit d'un portrait de groupe de hauts fonctionnaires bolchéviks, tous membres du conseil de tutelle de la construction. Six visages sur dix sont soigneusement noircis à l'encre. Les noms des disparus sont également caviardés. Le nom du disparu politique aussi bien que son visage sont deux signes d'identification qui permettent d'affirmer qu'il a bien existé. Privé du visage et du nom, il est réduit à l'état spectral. La photo suivante nous fait voir le suprême commandement de l'Armée Rouge ${ }^{12}$. Les taches d'encre semblent être des sacs noirs mis sur les têtes des maréchaux comme si, après leur écrasement physique, ces derniers étaient secondairement mis à mort par l'effacement de leurs traces sur la photo.

Sur une autre série de photos nous découvrons les visages noircis des chefs de l'une des républiques soviétiques ${ }^{13}$. Les photos étaient publiées dans une édition dessinée par Alexandre Rodtchenko. Après la condamnation des ces personnes, l'artiste a lui-même noirci leurs visages dans l'exemplaire qui lui appartenait. Si la zone noircie n'occupe qu'une partie sur les photos précédentes, elle couvre ici presque toute la surface de la page en la faisant ressembler d'une façon inattendue et frappante au Carré noir sur fond blanc de Malévitch. La ressemblance qui réunit une œuvre d'art et une "œuvre" étrange et terrifiante qui n'en est pas une, est plus que formelle. Dans les deux cas il s'agit des démarches iconoclastes. Si l'iconoclasme de Malévitch est une prise de position artistique, il n'en reste pas moins proche de l'iconoclasme involontaire de Rodtchenko, de cette "représentation négative» (die negative Darstellung) de la puissance (Macht) destructrice et inhumaine de l'État stalinien. Cette communauté rend difficile l'identification de la surface purement anti-représentative où triomphe le noir à un lieu d'« irruption » ou d'« indistinction » démocratique. En d'autres termes, elle met en question un certain paradigme intellectuel qui associe une fois pour toutes le radicalisme esthétique (dont Carré noir et Carré blanc sur fond blanc constituent deux extrémités) à la révolution politique. 
20 Pour mieux comprendre l'effet esthétique produit par une photo noircie, on peut analyser quelques exemples empruntés à la peinture. Les personnages d'un grand nombre de tableaux de Bruegel sont privés d'une partie du corps. Je ne retiens ici que deux exemples : Les Aveugles du musée Capodimonte et Les Mendiants (Les Estropiés) du Louvre. L'amputation des pieds ou des bras ainsi que la privation des yeux attaquent l'homme dans sa fonction prothétique en frappant sa vue ou son pouvoir de se déplacer (manipuler). Sur le plan esthétique, elle menace le personnage dans son humanité et fait sentir derrière l'humain qui lui est propre une masse informe et terrifiante de l'inhumain. Plus tard, sous l'influence de Bruegel, un effet de choc provoqué chez le spectateur par la vue des estropiés sera utilisé par Otto Dix dans un grand nombre de toiles dont Rue de Prague. Parmi les corps représentés sur le tableau il n'y a aucun qui soit intact: tous sont charcutés, partiels. Il ne s'agit pas seulement des corps des mutilés de guerre. On voit à l'arrière-plan des mannequins coupés au niveau de la poitrine et des prothèses de bras et de pieds exposées dans la vitrine. Les prothèses servent à remédier physiquement aux amputations et ne peuvent ni redonner au corps partiel son intégrité, ni restituer à l'homme son humanité. Du point de vue esthétique, elles inspirent de l'effroi car elles substituent assez brutalement une certaine unité biomécanique à une opposition traditionnelle et inébranlable entre l'homme et la machine.

21 Mais la déshumanisation de l'homme ayant pour cause une mutilation physique est moindre par rapport à la déshumanisation provoquée par la mutilation symbolique. Le dessin de Bruegel Apiculteurs construit sur l'effet de la dissimulation des visages, produit une très forte impression : les visages des personnages sont cachés derrière les masques d'osier. Comme les autres personnages de Bruegel, ceux des Apiculteurs ont subi une amputation, cette fois symbolique : ils sont privés de visage. À son tour, Otto Dix saisit l'affect en cachant les visages de ses personnages derrière les masques à gaz (série La guerre). Que ce soit chez Magritte, Francis Bacon ou Giacometti, l' « inquiétante étrangeté » de la tête sans visage apparaît régulièrement dans l'art occidental du $\mathrm{xx}^{\mathrm{e}}$ siècle.

22 La privation du visage est tout de même différente de la privation d'une partie du corps, d'un bras, d'un pied ou même des yeux. À strictement parler, le visage n'appartient pas au système du corps. Gilles Deleuze interprète le visage comme un système mur blanc (joue, front) / trou noir (yeux, bouche) en l'opposant au système du corps volume/cavité. Si la tête appartient au corps, le visage avec sa cartographie - les traits, les lignes, les rides - n'appartient pas au corps « même s'il s'applique et s'enroule sur un volume ${ }^{14} »$. C'est pourquoi la privation du visage qui sert de support à l'humanité dans l'homme, provoque chez le spectateur une angoisse inconsciente encore plus forte que l'amputation des membres. Elle fait pressentir (représente négativement) le trou noir de l'inhumain absolu ou de l' «informe » kantien. L'effroi et l'affect qui remplissent le spectateur en train de contempler les photos noircies s'expliquent par l'impossibilité d'identifier les visages disparus. L'informe de l'horreur vécue ne permet pas à la représentation de s'accomplir : le visage du personnage reste inconnu, le spectateur est impuissant à identifier la victime, son nom, les dates de naissance et de mort et - ce qui est aussi important - le lieu de sa mort. L'angoisse du spectateur est «sublime» mais ce sublime ne s'accompagne pas de plaisir comme c'était le cas chez Kant. 
23 À partir de là, il faut isoler le visage et la "visagéité » en général de l'idée de la production et du machinisme. Selon Deleuze, le visage est toujours inhumain, il est produit par une " machine abstraite ». Le concept de machine abstraite remplace dans la philosophie deleuzienne celui de «machines de désir », largement utilisé dans l'Anti๔dipe. La machine abstraite ne se limite pas à produire du désir sans sujet ; en mettant en forme le chaos initial, elle déclenche la «production » du visage. Deleuze cherche à séparer d'une part la visagéité et d'autre part le corps humain pour situer le visage du côté de l'inhumain, machinique, capitaliste. C'est par le visage que passe le racisme qui " propage les ondes du même jusqu'à l'extinction de ce qui ne se laisse pas identifier ${ }^{15}$ ». C'est pourquoi Deleuze attribue à l'art et à la littérature la tâche d'échapper au visage, défaire le visage en «traits de visagéité ». Cette analyse montre que le visage ne sera pas uniquement «le visage du despote » et que sa représentation sur une photo est parfois le seul moyen d'identifier la victime. Le visage, en tant que support identificatoire dans l'homme, ne peut pas être interprété seulement comme « figure du pouvoir ». Il faut séparer l'idée de la machine et l'idée du visage. On voit bien que l'effacement du visage est un phénomène totalitaire qui accompagne l'écrasement physique de l'opposant politique. Aussi Deleuze fait-il jouer l'opposition entre d'un côté le retour du même, sa répétition à l'infini et de l'autre côté un devenir vertigineux, un glissement dans un retour qui ne serait pas un retour au même (devenir femme, animal, fleur, etc.). Cette opposition est en dernière instance traduisible en opposition entre le paradigme technique ayant pour principe une certaine automation, un recommencement, un cycle et le paradigme organique particulièrement riche où toute similitude sur les lignes divergentes en devenir, par exemple l'œil du mollusque et l'œil de l'homme, s'explique par la défaillance dans le fonctionnement de la différence. Épouser l'opposition entre le technique et le biologique serait dresser une nouvelle fois l'opposition homme/machine et se montrer hostile aux objets techniques en supposant qu'ils « ne contiennent pas de réalité humaine ${ }^{16} »$. Dans ce cas, le degré de la technicité d'un objet est identifié au degré de son automatisme, à l'idée d'une répétition à l'identique qu'il comporte. Cette identification semble d'ailleurs fort problématique car, comme le montre Gilbert Simondon, l'automatisme est « un assez bas degré de perfection technique ${ }^{17}$ ». Celle-ci ne correspond pas au fonctionnement prédéterminé de la machine et sa fermeture sur elle-même mais, au contraire, à son ouverture, à ce que Simondon nomme "une marge d'indétermination " permettant à cet objet de briser son isolement. La signification qu'a l'automation est plutôt sociale et économique que technique. Ce n'est pas seulement le devenir biologique et le chaos (la "vie» deleuzienne, le retour de la différence) qu'on peut opposer à la répétition du même propre à la machine, mais aussi la représentation du trauma, son inscription par les intermédiaires techniques (les appareils) qui ont une marge d'indétermination plus large et qui véhiculent les processus de la création artistique.

Par conséquent, il faut situer la répétition propre à la machine non pas du côté du visage mais du côté de sa disparition. L'impossibilité d'identifier le visage du disparu sur une photo noircie provoque le retour du trauma. Ce retour du même ayant pour cause un "détraquement" initial renvoie au modèle machinique de l'inconscient élaboré par Deleuze. Si les machines décrites dans l'Anti-〔Edipe sont des machines complexes et ont un assez bas degré d'automatisme (elles sont sociales avant d'être techniques) le concept de machine fait néanmoins appel à la répétition et l'automatisme de l'expression, en s'opposant à la répétition par retour de la différence, décrite dans Différence et répétition. Ayant pour principe la répétition du même, la 
machine privilégie le cycle. Elle met en forme la matière, ne rompt pas avec le schéma hylémorphique et peut aller jusqu'au bout de l'idée capitaliste de la production. Mais cette productivité renvoie à un détraquement initial, à l'impossibilité d'articuler un trauma. La reprise infinie de la représentation, propre au machinisme, est une forme revêtue par la reproduction incessante du même. Elle s'explique par un reste "sublime " résultant de l'impossibilité d'inscrire une expérience de choc (Freud, Par delà le principe de plaisir). L'état du détraquement est donc essentiel pour les machines : les machines « ne marchent que détraquées ${ }^{18}$ ".

Or, le visage - à condition qu'il soit saisi, représenté par des intermédiaires techniques que sont les appareils - n'est pas une figure du pouvoir et n'est pas produit par les machines, pas plus qu'il serait politiquement neutre. S'inscrivant dans la tradition qui vient des portraits de groupe dans la peinture hollandaise, les portraits photographiques sont loin d'être de simples procédés mnémoniques. La réunion dans le même espace photographique des visages de ceux qui partagent les mêmes idées fait exister politiquement un groupe social - qu'il s'agisse de leaders politiques, de travailleurs ou d'un groupe de riches bourgeois bienfaiteurs comme ceux qui figurent sur une photo trouvée dans les archives de Nijni-Novgorod ${ }^{19}$.

Figure 3

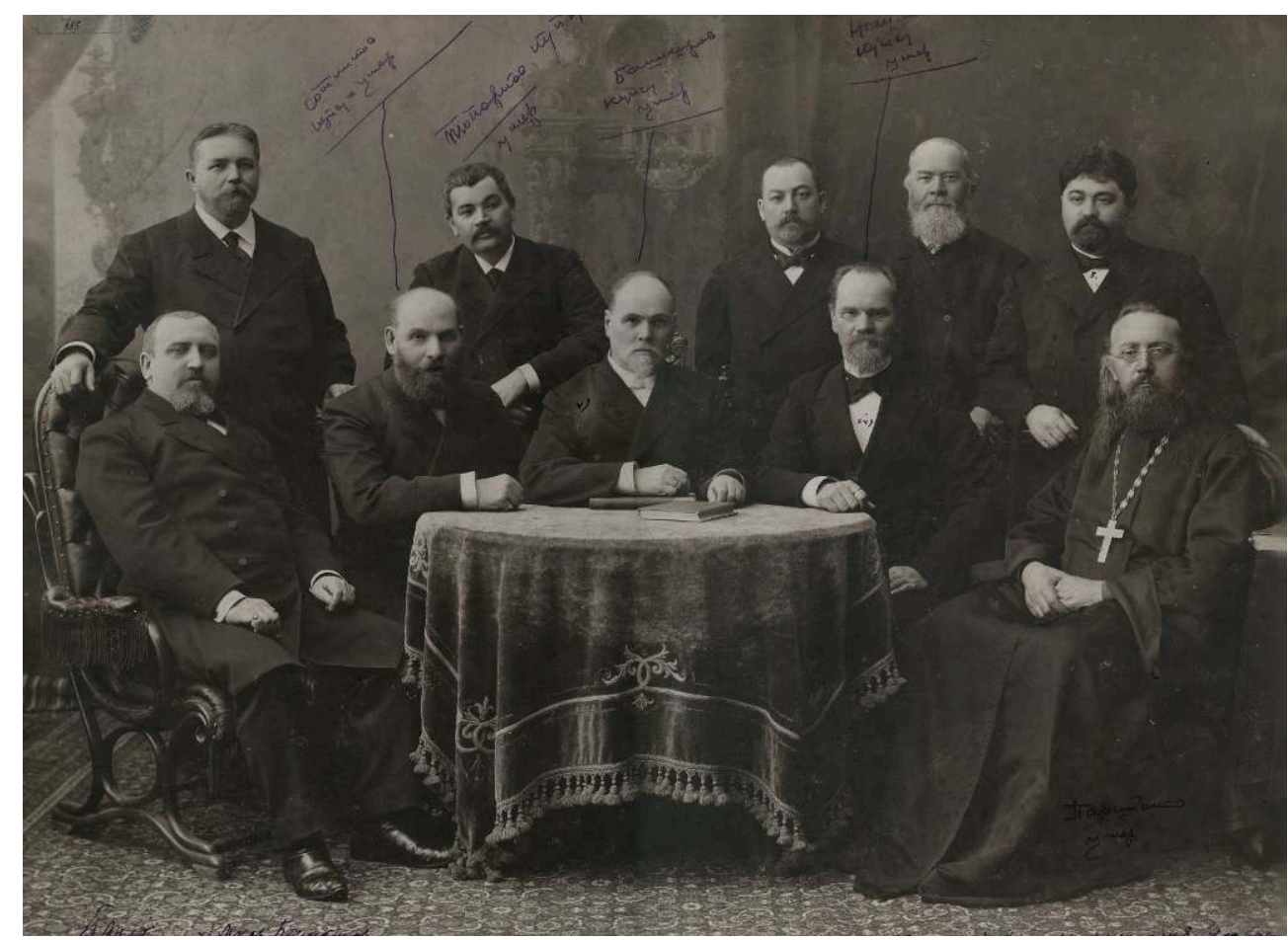

Dans les années 1930, elle a attiré l'attention des gens du NKVD (police secrète rebaptisé plus tard «KGB »). L'intérêt de ces photos consiste à porter des inscriptions, les traces du « travail » des enquêteurs du NKVD qui les utilisaient comme preuves pour identifier les notables de l'ancien régime et les inscrire sur les listes des suspects à passer par les armes. C'est de la même façon qu'ils se sont servis de deux autres photos prises peu avant le Révolution et représentant les policiers ${ }^{20}$. 
Figure 4

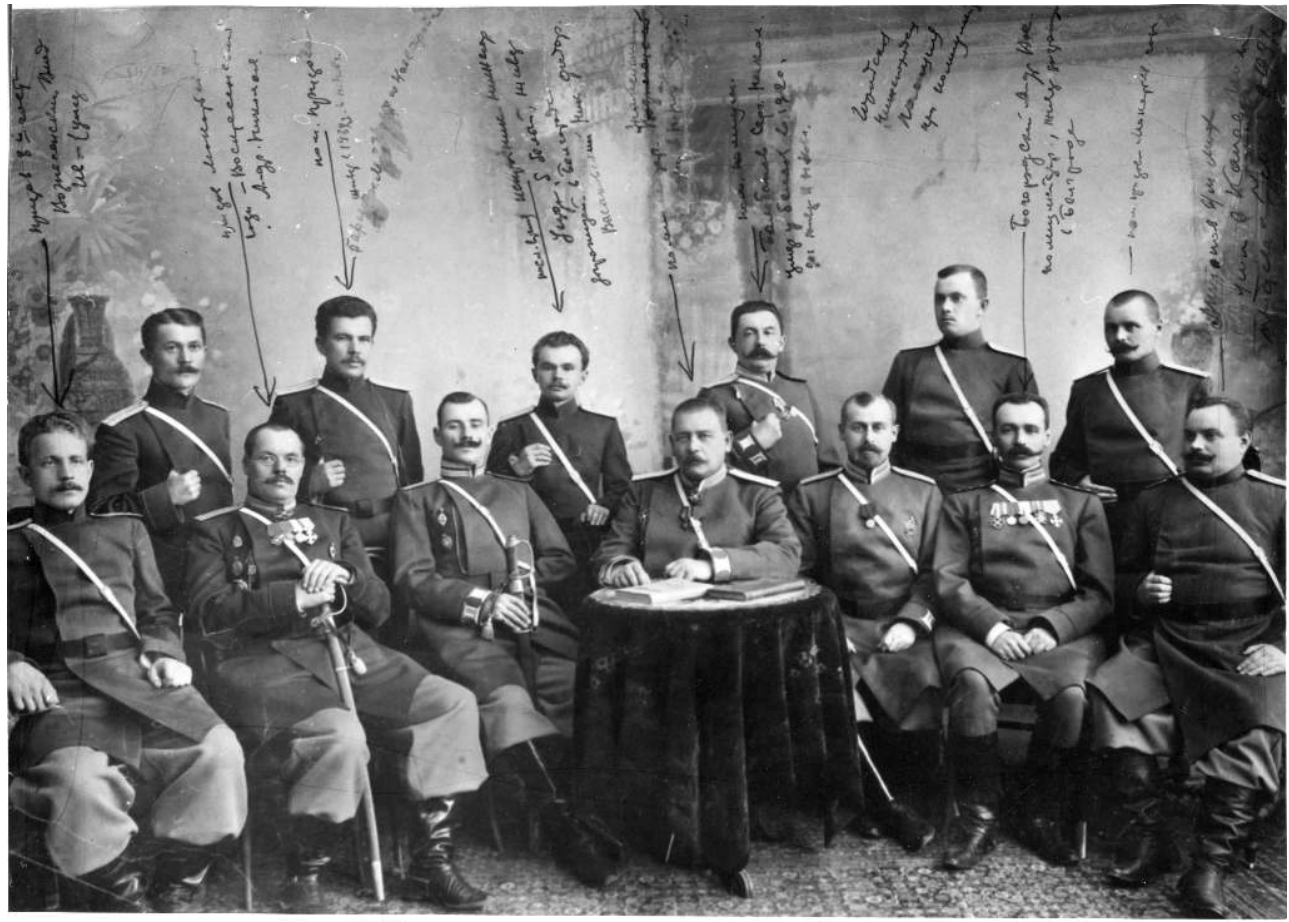

Figure 5

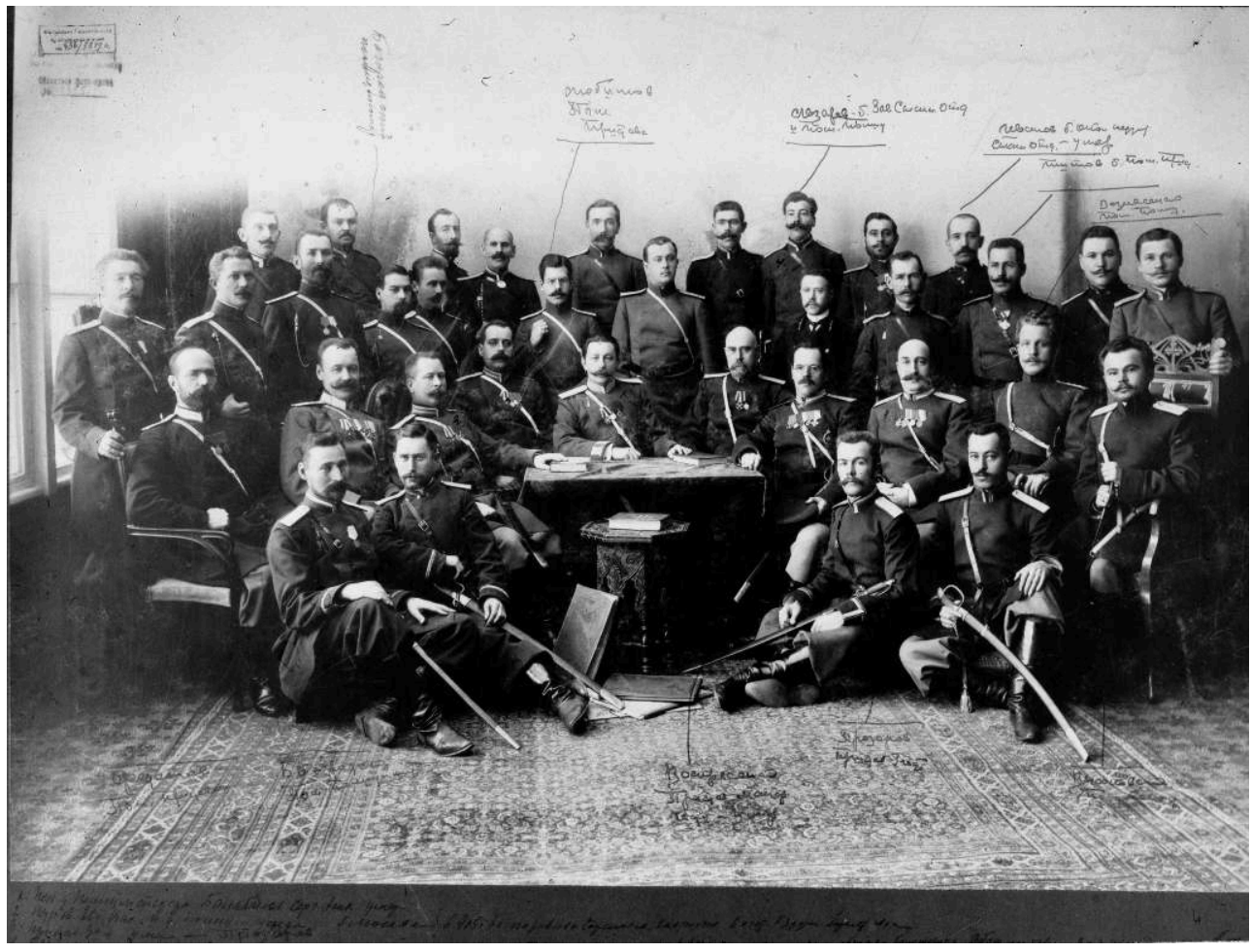

Le nom et le visage de la victime sont rayés de l'histoire. Mais cet oubli est fréquemment suivi de ce qu'on peut nommer, à la suite de Lyotard, « l'oubli de l'oubli ». C'est à ce titre qu'on peut considérer la tâche de combler les lacunes dans l'histoire du Parti en complétant les archives par les copies souvent fragmentaires des photos échappées au caviardage. Malgré l'apparence, ce processus est beaucoup moins une 
stratégie de réhabilitation que la tentative d'échapper à l'histoire, d'oublier, de faire comme si la terreur n'avait pas eu lieu. Il ne s'agissait pas de représenter cet événement politique «informe " mais de le refouler. Parallèlement, les photos noircies étaient retirées des archives car elles étaient classifiées « abîmées ». Ainsi, la photo ci-dessous, trouvée dans les archives du Parti à Nijni-Novgorod, est brutalement coupée de trois côtés ${ }^{21}$.

Figure 6

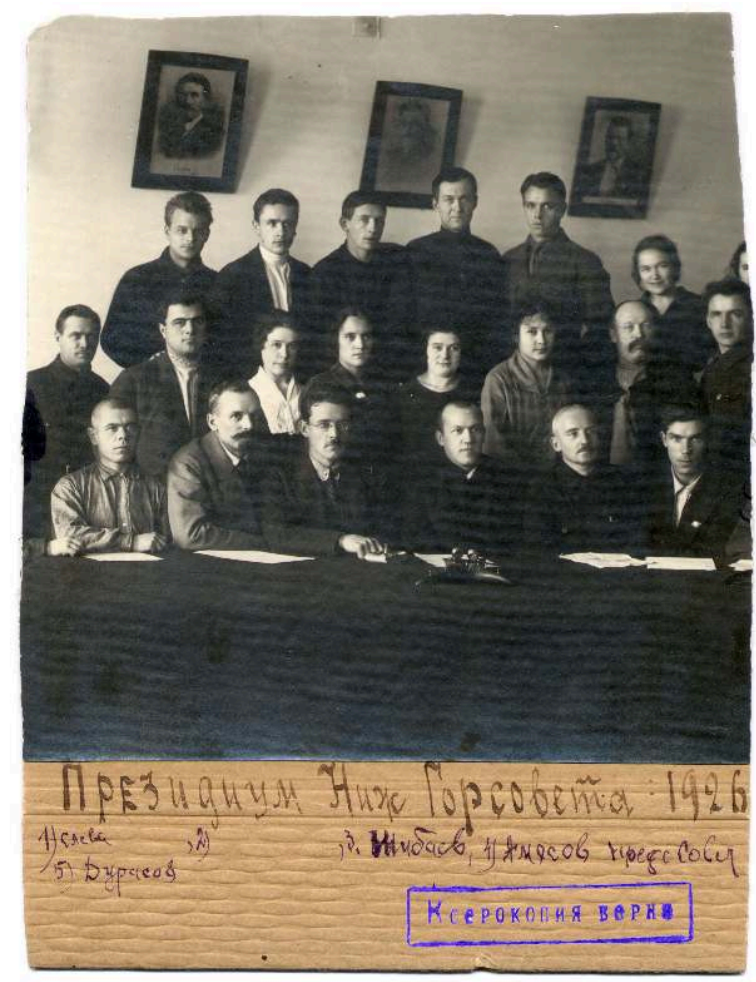

À gauche et à droite on voit les parties du corps qui appartiennent aux personnages supprimés. Mais avant de recevoir un coup de ciseaux, cette photo a été noircie selon la méthode en usage dans les années 1930 : à la regarder de plus près, nous découvrons de deux côtés des traces d'encre noire. La fiche d'archive dit que la photo est passée par une restauration. Il est probable que cette soi-disant restauration consistait à éliminer les parties noircies, ce qui était une façon de se débarrasser des traces laissées par le choc des années 1930 (il est d'ailleurs également possible que la personne qui a noirci la photo, ait jugé cela insuffisant et ait coupé par la suite les représentations des victimes). Si, dans un premier temps, la disparition politique s'accompagnait de l'effacement de l'image du disparu, c'était plus tard cet effacement qu'on voulait rayer de la mémoire collective. Les traces de cet effacement sont parfois effacées au sens strict, comme on le voit sur une autre photo trouvée dans les archives du Parti à NijniNovgorod. 


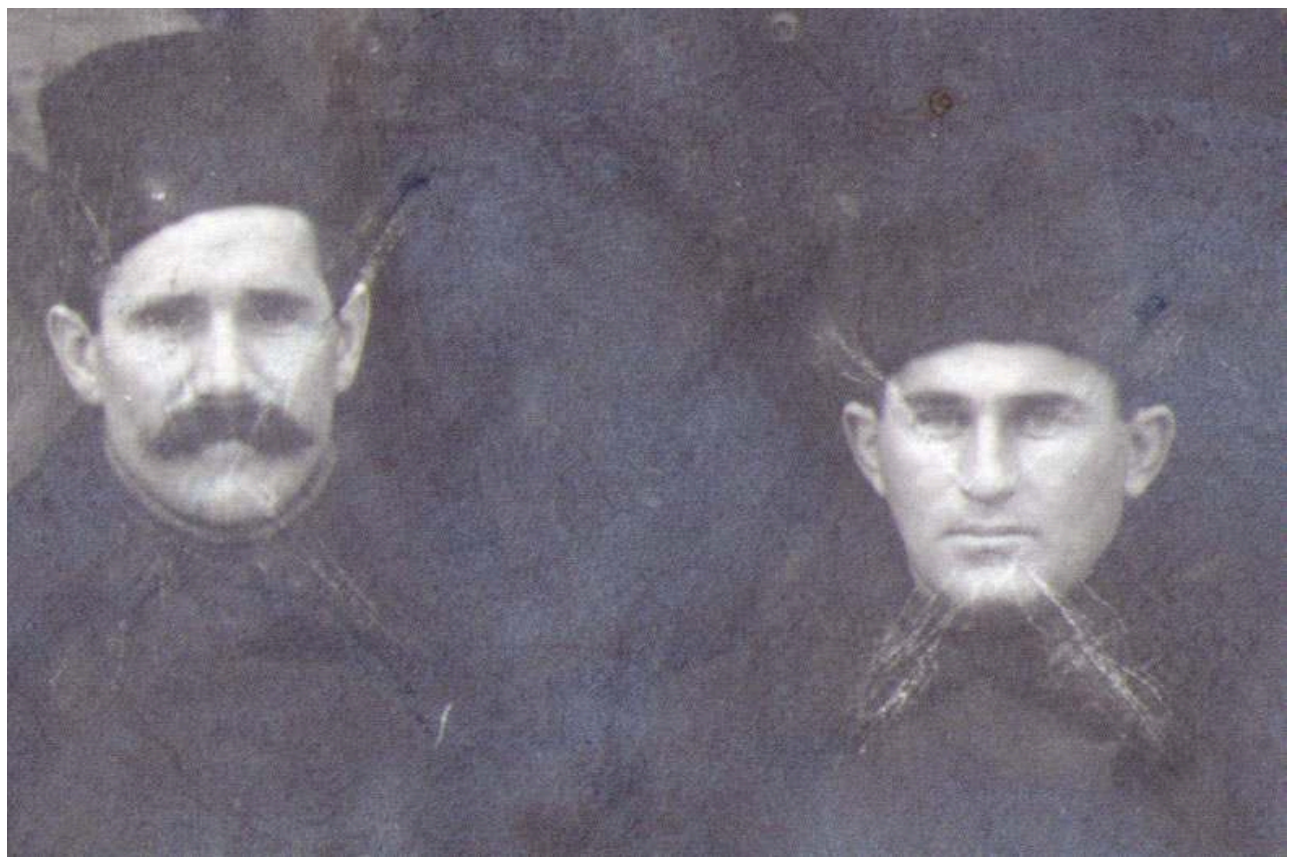

Nous découvrons les traces d'un travail méticuleux que quelqu'un a fait pour gommer les deux traits en croix sur les visages de deux personnes au centre ${ }^{22}$. Rayer en croix les visages des ennemis du peuple était, à l'époque stalinienne, l'autre forme - moins radicale que le caviardage - de leur exécution symbolique. On peut la voir sur une autre photo représentant les délégués communistes de Nijni-Novgorod en compagnie de Staline $^{23}$.

Figure 8

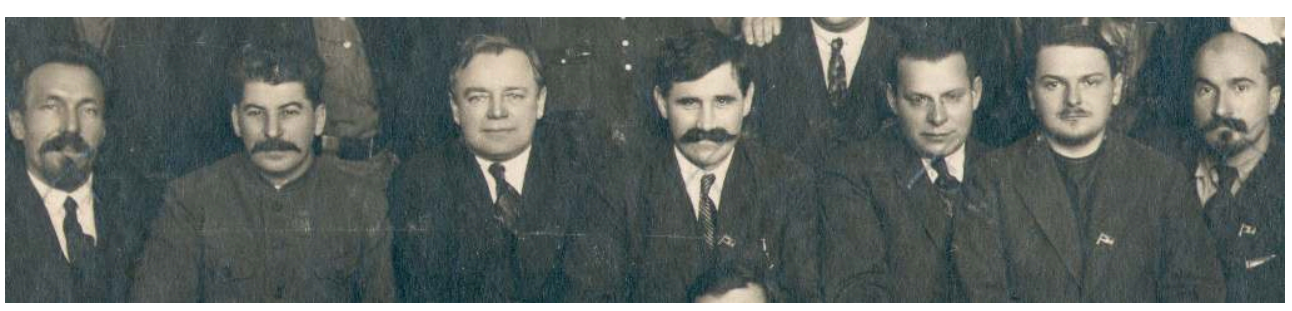

Huit visages sur trente sont rayés en croix. Souvent, on rayait les photos dans un premier temps, avant de les noircir et nous découvrons les rayures en croix sous une couche d'encre.

31 La recherche de pareilles photos nous met face à un problème : le plus souvent nous ne trouvons que des preuves indirectes au fait qu'une photo, après avoir subi un coup de ciseaux ou de crayon, est passée par une restauration. Les traces de l'attentat qu'on a essayé de cacher restent imperceptibles pour un coup d'œil macroscopique. On ne les découvre que si l'œil examine spécialement la surface de la photo ou, s'il s'agit de sa copie numérique, à condition que son agrandissement soit considérable.

La question qu'il faut poser est la suivante : l'effacement des traces du disparu est-il définitif? Sera-t-il possible de lui rendre son visage, de reconstituer les traces photographiques comme on reconstitue un palimpseste? 

de Staline par le peintre Brodsky était largement diffusé par la propagande soviétique. Ce portait était fait d'après une photo où Staline était représenté en compagnie de Kirov, assassiné, comme le supposent les historiens, par l'ordre du dictateur. Mais la photo dont il s'agit n'est elle-même pas authentique. Elle existe dans une autre version où les personnages sont trois. Cette variante n'est pourtant pas authentique non plus. Une version plus complète nous fait voir à côté de Staline encore une personne : il s'agit de Nicolas Antipov arrêté et fusillé en 1938. Cette version était à son tour considérée comme authentique jusqu'à ce qu'on en ait découvert une autre où les personnages sont $\mathrm{cinq}^{24}$. On ne peut pas dire que la vérité historique recule lors de la reconstitution de cette photo ou qu'elle nous est interdite par un reste irreprésentable, impossible à exprimer en aucun cas. Au contraire, chaque photo réapparue nous fait approcher de la vérité. Cela signifie que malgré les difficultés, la reconstitution des photos falsifiées ou noircies aboutira à la représentation du sublime historique. Cette absorption par représentation sera différente de la situation où la psyché se montre incapable de dépasser un choc sensible. Dans Par delà le principe de plaisir Freud décrit le jeu de la bobine auquel se livrait son petit fils lors des absences provisoires de sa mère. Comme l'explique Jean-Louis Déotte, ce jeu constitue un appareillage élémentaire qui transforme l'énergie libidinale de l'état libre à l'état lié et permet à l'enfant de faire face à une expérience traumatisante ${ }^{25}$. C'est sur ce modèle qu'on peut concevoir la représentation de l'expérience sublime qui peut être exprimée à condition de l'invention ou du retour des appareils, à condition qu'on redécouvre une photo authentique. Il faut rendre les cadres du "régime esthétique» aussi larges qu'auparavant pour que le banni et le refoulé y puisse réapparaître. La découverte du visage disparu met fin à la tâche de vouer l'irreprésentable à la répétition à l'infini. Il y a toujours la probabilité que la version dont nous disposons ne soit pas authentique, qu'il y en ait d'autres, plus complètes. Mais il n'y a pas de doute que la représentation peut progresser, que le nombre des falsifications n'est pas illimité, qu'on finit tôt ou tard par retrouver la version originale ou du moins les traces de celle-ci. Le sublime - le sublime d'appareils -, sera mis en paroles ou images. C'est donc la première conséquence qu'on peut tirer de l'analyse des photos en question : la représentation du sublime est possible et elle se trouve à condition de découvrir la photo authentique ou du moins ses traces.

En même temps, on voit que la représentation doit remédier aux difficultés d'ordre esthético-politique. Si les cadres du régime esthétique sont assez larges pour que toute expérience puisse s'y inscrire, la «convenance » entre la forme et le contenu peut néanmoins déraper en cas du caviardage ou de la falsification de telle ou telle photo, en cas d'attentat contre une transparence représentative qui s'est instaurée avec l'invention de l'appareil photographique. La représentation peut avoir des étapes qui sont comme autant de cercles vicieux. Cette représentation par étapes a quelque chose de machinique, elle est proche de la répétition du même, de l'obsession se renouvelant toujours provoquée par un trauma. Si une version nouvellement découverte s'écarte de la version authentique à découvrir, c'est qu'elle a son seuil représentatif. En même temps, même s'il peut avoir une dimension prothétique ou machinique - la représentation qui n'aboutit que partiellement, le retour cyclique du même propre à la machine - c'est l'appareil qui rend la représentation possible. Chaque photo nouvellement découverte fait élargir les «cercles» de représentation. L'apparition 
d'un nouveau personnage sur la photo correspond à un degré de plus dans le dévoilement de la vérité.

Le « trou noir » qui fait obstacle à l'identification du disparu et à la représentation de sa disparition n'affecte pas seulement la personnalité de ce dernier. Toute disparition politique (arrestation) a pour résultat un effet de sublimité qui dissout la synthèse hylémorphique. Elle provoque de l'angoisse dans l'entourage du disparu car elle attaque son « milieu associé » (Simondon). Les photos des fusillés n'étaient pas noircies par les censeurs ni par les gens du NKVD. C'étaient les collègues, les amis et même les épouses ou enfants des victimes qui, saisis de peur, faisait disparaitre les visages de leurs proches. Cette action qui nous choque et qui paraît incroyable avait pourtant une raison simple: les photos des ennemis du peuple pouvaient être utilisées comme preuves à charge contre ceux qui les gardaient.

Même aujourd'hui la recherche et la publication de telles photos n'est pas sans obstacles. Les familles qui ont dans leur album les photos aux visages noircis ne veulent pas les montrer aux étrangers : jusqu'à présent, l'effort de représenter ce qui est arrivé est perçu comme douloureux. Mais le paradoxe est que ce trauma doit être articulé.

La deuxième conséquence qu'on peut tirer de notre analyse est la suivante : même dans le « régime esthétique des arts » (Rancière) la représentation peut être contrariée, elle n'est pas sans difficulté sur le plan technique et politique. Si la représentation du sublime est possible dans un grand nombre de cas, elle est souvent différée par des mesures antireprésentatives ("policières », en termes de Rancière), en l'occurrence, par la privation du visage que subit «l'ennemi du peuple». Rancière attribue à la photographie un rôle de moyen technique au service des régimes esthétiques. Mais la photographie n'est pas en elle-même politiquement neutre, elle n'est pas une simple "prothèse " qui prolonge les capacités de l'homme tout en restant extérieur à son corps. Ayant une valeur de preuve, la photographie est un appareil qui inscrit une expérience de choc, qu'il s'agisse d'une expérience historique ou individuelle.

\section{NOTES}

1. Kant, Kritik der Urteilskraft, S. 50. Quellen Philosophie: Deutscher Idealismus, S. 2562 (vgl. KantW Bd. 10, S. 103).

2. Kant, Kritik der Urteilskraft, S. 192. Quellen Philosophie: Deutscher Idealismus, S. 2704 (vgl. Kant-W Bd. 10, S. 201).

3. Jean-François Lyotard, L'enthousiasme. La critique kantienne de l'histoire, Paris, Galilée, 1986. JeanFrançois Lyotard, Cinq leçons sur l'analitique du sublime, (Kant, Critique de la faculté de juger, § 23-29), Paris, Galilée, 1991. Jean-François Lyotard, Inhumain, Paris, Galilée, 1988. Jean-François Lyotard, Misère de la philosophie, Paris, Galilée, 2000. Jean-François Lyotard, Judicieux dans le différend, in La faculté de juger, Paris, Minuit, 1985.

4. Jacques Rancière, Malaise dans l'esthétique, Paris, Galilée, 2004. Jacques Rancière, Le destin des images, Paris, La fabrique, 2003. Jacques Rancière, La Mésentente, Paris, Galilée, 1995.

5. Jacques Rancière. Le destin des images, Paris, La fabrique, 2003, p. 38

6. Ibid, p. 90 
7. Jean-François Lyotard, Discours, figure, Paris, Klincksieck, 2002.

8. Jacques Rancière, Le destin des images, Paris, La fabrique, 2003.

9. Jean-Louis Déotte et Alain Brossat (dir.), La mort dissoute. Disparition et spectralité, Paris, L'Harmattan, 2002.

10. Archives audiovisuelles de Nijni-Novgorod // Album № 12, «Construction routière dans la région de Gorky («Kraïdortrans»). Photomontage d'A. Ivanov Allocutions de J. Kaganovitch et E. Pramnek au premier congrès des constructeurs de route. Portraits de travailleurs de choc, le 22 mai 1934.

11. Archives audiovisuelles de Nijni-Novgorod // Album № 7, « Le pont Nikolas Pachomov traversant l'Oka à Gorky. 1930-1933 ». 1. Le portrait de groupe des travailleurs d'honneur du chantier : A. Jdanov, J. Kaganovitch, A. Gratchev, M. Doubnov. 29/IV1933. Photo DTK, Gorky.

12. David Kind, Le commissaire disparaît. La falsification des photographies et des ceuvres d'art dans la Russie de Staline, Calmann-Levy, 2005.

13. David Kind, Le commissaire disparait.

14. Gilles Deleuze, Félix Guattari, Capitalisme et schizophrénie 2, Mille plateaux, Paris, Minuit, 1980. p. 208.

15. Ibid., p. 218.

16. Gilbert Simondon, Du mode d'existence des objets techniques, Paris, Aubier, 1989, p. 9.

17. Ibid, p. 11.

18. Gilles Deleuze, Félix Guattari, Capitalisme et schizophrénie 1. Anti-〔Edipe, Paris, Minuit, 1972, p. 38-39.

19. Archives audiovisuelles de Nijni-Novgorod // Inventaire 1.1/8, 66-3060. L'archevêque Nazaire et le groupe des bienfaiteurs de l'orphelinat Kutaissov. 1913.

20. Archives audiovisuelles de Nijni-Novgorod // Un groupe de policiers de Nijni-Novgorod avec le chef de police A. Znamenski. Inventaire 726.; Archives audiovisuelles de Nijni-Novgorod // Police. Deux groupes. Inventaire 300.

21. Archives du Comité régional du Parti Communiste de Nijni-Novgorod // La présidence du Conseil municipal de Nijni-Novgorod: Durassov, Amossov, Chibaev. Nijni-Novgorod, 1926. Fonds 7853, dossier 387, inventaire1.

22. Archives du Comité régional du Parti Communiste de Nijni-Novgorod // Les délégués de Nijni-Nogorod au Xe congrès des Conseils près de l'entrée du Théâtre Bolchoï: Machotine, I. Tchugurine, N. Uglanov, Kaganovitch, A.Taganov. Le 23 décembre 1930 г. Fonds 7853, dossier 418, inventaire 1.

23. Archives du Comité régional du Parti Communiste de Nijni-Novgorod // Le $x v^{e}$ congrès du VKP(b). La délégation de Nijni-Novgorod avec les membres du gouvernement. Moscou, le 15 mars 1927. Fonds 7853, dossier 394, inventaire 1.

24. David Kind, Le commissaire disparaît. La falsification des photographies et des ceuvres d'art dans la Russie de Staline, Paris, Calmann-Levy, 2005.

25. Jean-Louis Déotte, La vidéo à l'épreuve de la disparition (J. Hanono, F. Menini), in Intermédialité, $\mathrm{n}^{\circ} 10,2007$. 


\section{RÉSUMÉS}

Cet article est consacré à la polémique Jacques Rancière / Jean-François Lyotard autour de la notion de sublime chez Kant. Bien entendu cette controverse au sujet du sublime est loin d'être un simple désaccord exégétique. L'Analytique du sublime constitue un « champ de bataille » entre deux points de vue diamétralement opposés sur l'esthétique et la politique : le beau et le sublime, l'œuvre d'art et son support, l'émancipation et l'identité. À partir de l'analyse de quelques photographies et quelques exemples tirés de la peinture, cet article se propose d'interroger la pertinence de la notion de sublime pour la description de certains phénomènes esthétiques et politiques du siècle passé.

\section{AUTEUR}

\section{DENIS SKOPIN}

Université Dobrolubov de Nijni-Novgorod 